\title{
Predictive correlations for leaking heat transfer fluid aerosols in air
}

\author{
K. Krishna ${ }^{\text {ab }}$, T.K. Kim ${ }^{\text {ac }}$, K.D. Kihm ${ }^{\text {ac }}$, W.J. Rogers ${ }^{\text {ab }}$, M.S. Mannan ${ }^{\text {ab,* }}$ \\ a Mary Kay O'Connor Process Safety Center, Texas A\&M University, College Station, TX 77843-3122, USA \\ ${ }^{\mathrm{b}}$ Chemical Engineering Department, Texas A\&M University, College Station, TX 77843-3122, USA \\ ${ }^{c}$ Mechanical Engineering Department, Texas A\&M University, College Station, TX 77843-3123, USA
}

\begin{abstract}
Mist or aerosol explosions present a serious hazard to process industries. Heat transfer fluids are widely used in the chemical process industry, are flammable above their flash points, and can cause aerosol explosions. Though the possibility of aerosol explosions has been widely documented, knowledge about their explosive potential is limited. Studying the formation of such aerosols by emulating leaks in process equipment will help define a source term for aerosol dispersions and aid in characterizing their explosion hazards.

Current research conducted at the Mary Kay O'Connor Process Safety Center involves the non-intrusive measurement of heat transfer fluid aerosol sprays using a Malvern Diffraction Particle Analyzer. A predictive correlation relating aerosol droplet diameters to bulk liquid pressures, temperatures, thermal and fluid properties, leak sizes, and ambient conditions is presented. This correlation can be used to predict the conditions under which leaks will result in the formation of aerosols and ultimately help in estimating the explosion hazards of heat transfer fluid aerosols. The goal is to provide information that will help improve safety in process industries.
\end{abstract}

(C) 2003 Elsevier Science Ltd. All rights reserved.

Keywords: Aerosol; Spray; Heat transfer fluid; Drop sizing; Malvern Laser Diffraction Particle Analyzer; Correlation

\section{Introduction}

Incidents in the chemical industry quite often result in the loss of containment. Escaping fluids are released into the surrounding atmosphere in the form of a liquid or vapor or both. Liquid releases, depending on the conditions, may atomize to form an aerosol, which is a dispersion of liquid droplets in air. These droplets have the potential to disperse over a significantly larger area than the bulk liquid. It is a common misconception that combustible liquids are safe below their flash points (Febo \& Valiulis, 1995), but aerosols of combustible liquids at temperatures well below their flash points can be as explosive as vapor-air mixtures (Eichhorn, 1955; Bowen \& Shirvill, 1994).

\footnotetext{
* Corresponding author. Tel.: +1-979-845-3489; fax: +1-979-4581493.

Abbreviations:

AAR: alkylated aromatic; MTP: modified terphenyls; DTA: di/triaryls; DAA: di-aryl alkyl; WMO: white mineral oil; BDO: biphenyl/diphenyl oxide

E-mail address: mannan@tamu.edu (M.S. Mannan).
}

Heat transfer fluids (HTFs) are high flash point synthetic liquids, which are used over4 wide ranges of conditions in the chemical process industry and are available in a wide range of properties. HTFs are generally considered benign below their flash points, but they are used at high pressures and have the potential to form aerosols upon leaking (Vincent \& Howard, 1976).

The main processes associated with the formation of aerosols from leaks are not well documented, and there is a lack of knowledge about droplet diameters that result from a leakage under various conditions. This information is critically needed for aerosol flammability studies and for development of preventative measures to improve safety in the process industry.

\section{Experimental methodology}

The objective of this research is to study the atomization characteristics of heat transfer fluids. The emphasis is on formation distances and drop size distributions of aerosols created by plain orifice atomization to emulate a leak in a process system. An experimental 
approach employs the Malvern Laser Diffraction Particle Analyzer (Malvern laser) for a non-intrusive analysis technique.

Fig. 1 shows a schematic of the experimental equipment, which consists of a pressurized fluid cell and delivery system, a spray collection and exhaust system, and the Malvern laser. The fluid cell is a 5.9 liter aluminum cylinder with an internal diameter of $17 \mathrm{~cm}$ and a height of $36 \mathrm{~cm}$. To simulate leaks, orifices are drilled into brass plugs. The positioning system consists of two precision rails for the $\mathrm{X}$ direction along the centerline of the spray from the orifice and the $\mathrm{Y}$ or radial direction perpendicular to the $\mathrm{X}$ direction. The exhaust system includes a collection chamber for the spray, a mist separator that removes aerosol larger than 5 micron in diameter, and an explosion proof blower to remove the vapor and aerosol phases.

The Malvern laser consists of a $2 \mathrm{~mW}$ Helium-Neon laser tube and a ring diode detector. The laser light at a wavelength of $632.8 \mathrm{~nm}$ is a parallel beam with a diameter of $1.8 \mathrm{~mm}$ (Barth, 1984). When the aerosol droplets pass through this beam, they diffract the light by amounts inversely proportional to the droplet diameter (Hecht, 2002). This diffracted light falls on 31 concentric ring diodes in the detector with each ring detecting a certain diameter range of droplets. The light intensities on each of these diodes are converted into mean droplet diameter data by a computer. The Malvern laser measurement technique for aerosols has been widely studied in the area of automotive fuel spray combustion (Kihm, Terracina, Payne, \& Caton, 1994). Sukmarg, Krishna, Rogers, Kihm, \& Mannan. (2002) have provided additional information.

A HTF in the fluid cell is heated to the required temperature using heating tape and is pressurized with nitrogen gas to propel it through the orifice. Measurement of the HTF temperature and pressure are made as close to the orifice as possible. Upon pressurization the heated

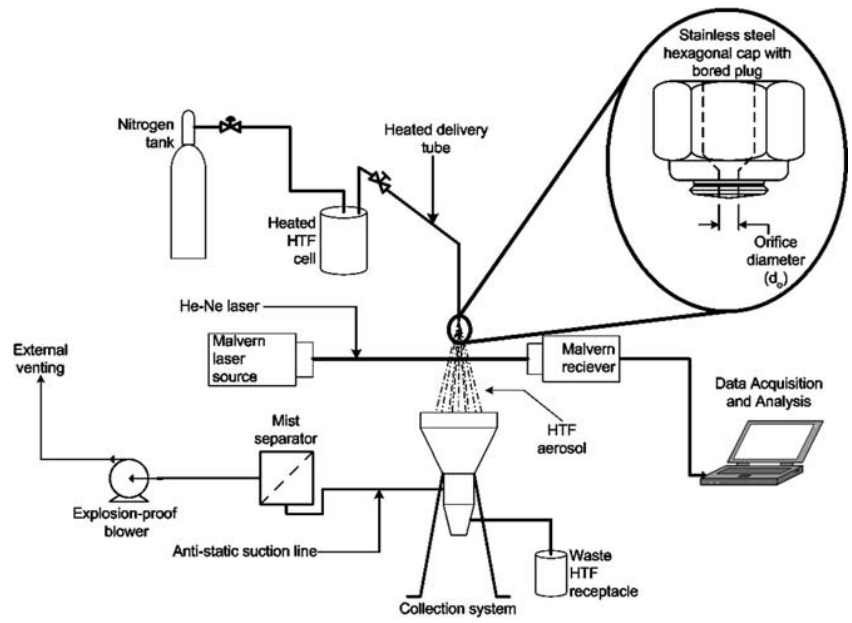

Fig. 1. Aerosol measurement apparatus.
HTF is sprayed through the laser beam and into the collection chamber. Each drop size distribution measurement is an average of data from 500 diode sweeps. The uncertainty in the measurement of aerosol droplet diameters by the Malvern is estimated to be \pm 5 micron, which combines the Malvern laser instrumentation contribution and the limitations of the diffraction technique. Additionally, the Malvern laser system was calibrated within $\pm 3 \%$ using a standard reticle. Hence the total error for spherical droplets over a measurement range of 0 100 microns is within \pm 8 microns.

Photographs of the sprays at various conditions help to interpret the non-spherical droplet size data in the transition regions (Sukmarg et al., 2002), where the stream atomizes into aerosol. In these regions the Malvern laser reports mean droplet diameters with a higher uncertainty because the Malvern laser assumes the droplets are spherical.

\section{Experimental observations}

The focus of this research was to emulate the formation of an aerosol from a HTF leak in an industrial process and to study the effects of process operating conditions, temperature and pressure, and leak sizes. A summary of the tested conditions for each fluid is presented in Fig. 2. At higher temperatures, liquids have lower densities, lower viscosities, and lower surface tensions and therefore form aerosols with smaller mean

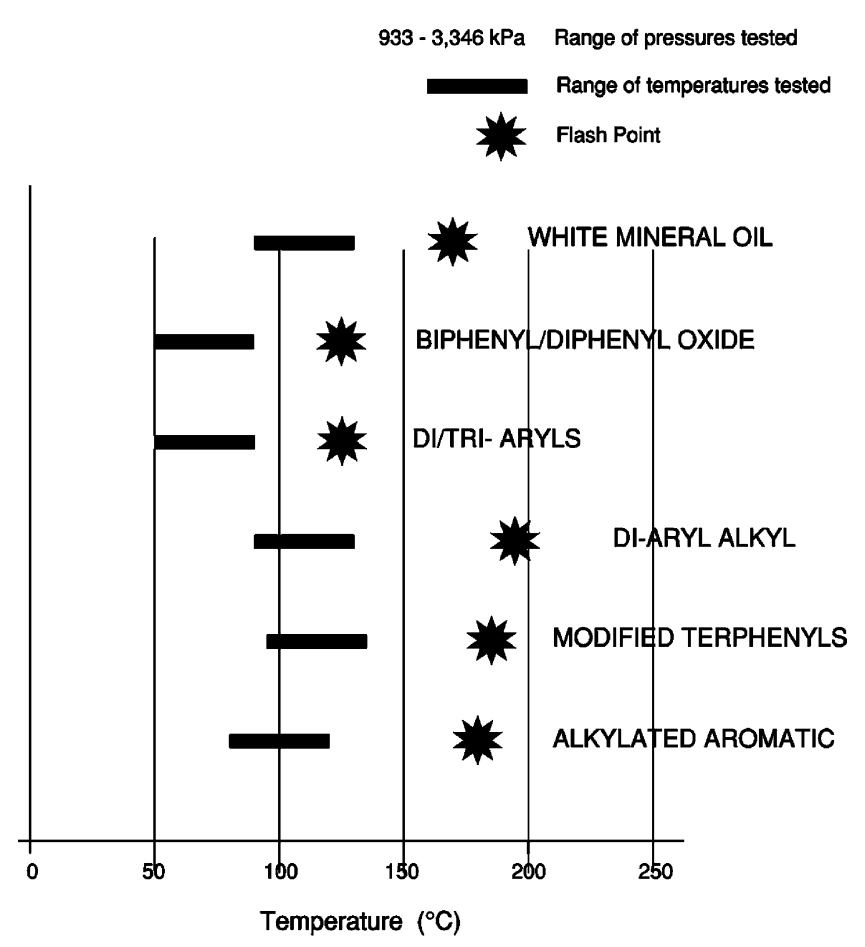

Fig. 2. Summary of the experimental conditions and flash points of the fluids tested. 


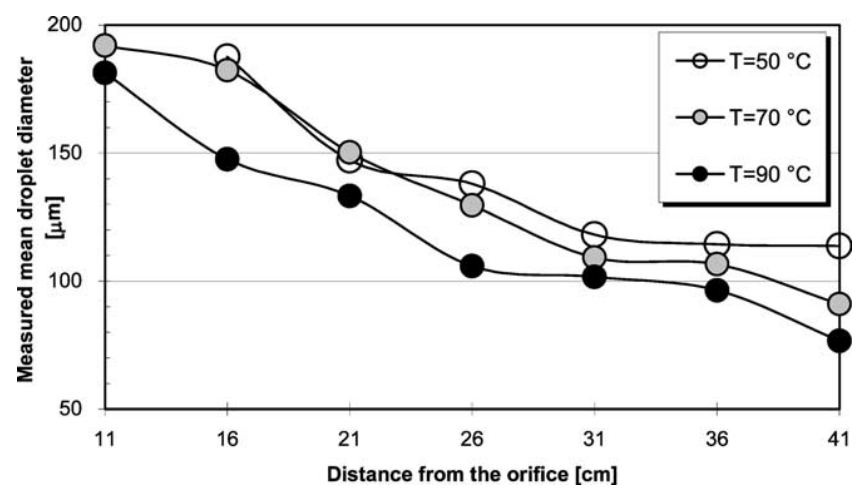

Fig. 3. The effect of temperature on the atomization of BDO (biphenyl/diphenyl oxide), under a pressure of $1967 \mathrm{kPa}$ and through an orifice of diameter $0.38 \mathrm{~mm}$.

droplet diameters, as shown in Fig. 3. Pressure has a more direct influence on the atomization. Higher injection pressures increase the liquid velocity, which shortens the aerosol formation distances and also produces smaller mean droplet diameters, as shown in Fig. 4.

\section{HTF physical properties}

In order to analyze the experimental data, HTF physical properties of density, viscosity, and surface tension as functions of temperature, were needed. Some data was obtained from the manufacturers, but in cases where data was insufficient, measurements were made to obtain it. Correlations were then fitted to the data to allow interpolation of the data for the dimensionless modeling.

Correlations of density and viscosity variation with temperature, derived from data provided by the manu-
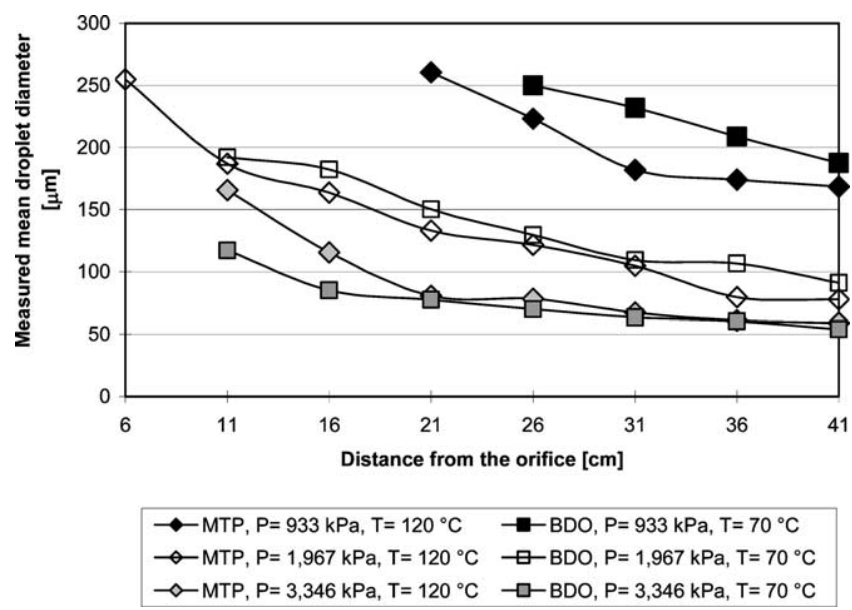

Fig. 4. The effect of injection pressure on the atomization of two heat transfer fluids through an orifice of diameter $0.38 \mathrm{~mm}$. MTP: modified terphenyls $\left(\mathrm{T}=120^{\circ} \mathrm{C}\right)$; BDO:-biphenyl/diphenyl oxide $\left(\mathrm{T}=70^{\circ} \mathrm{C}\right)$.

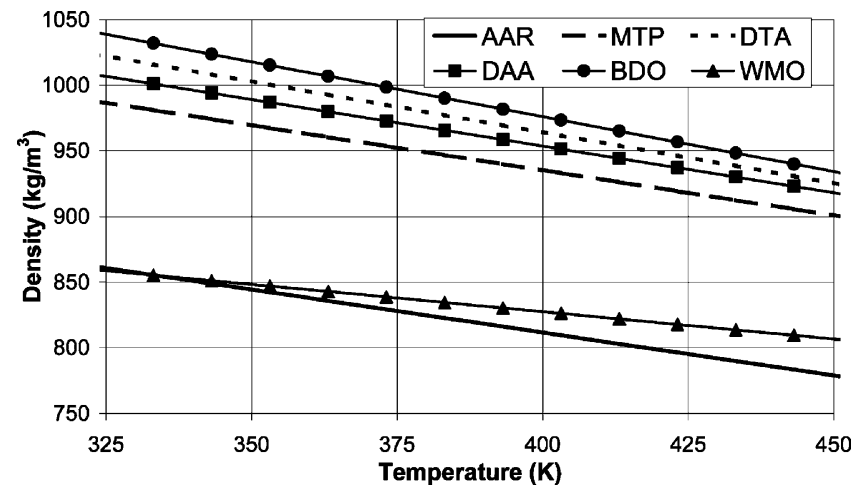

Fig. 5. Variation of density with temperature for the six HTFs.

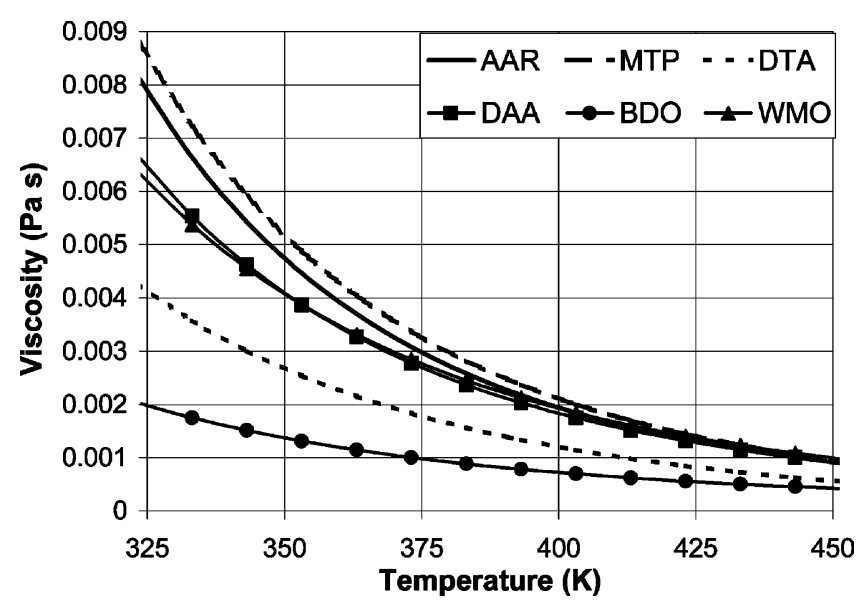

Fig. 6. Variation of viscosity with temperature for the six HTFs.

facturers, are shown in Figs. 5 and 6, respectively. Surface tension of the six HTFs was measured using a Fisher Surface Tensiomat, Model 21, which operates according to the ASTM D-971 and D1331 methods. The overall error for surface tension measurements of $-3.5 \%$ to $-6.5 \%$ consists of the error due to calibration $( \pm 1.5 \%)$ and the maximum error obtained from verification with water $(-5.5 \%)$. Fig. 7 displays a correlation of surface

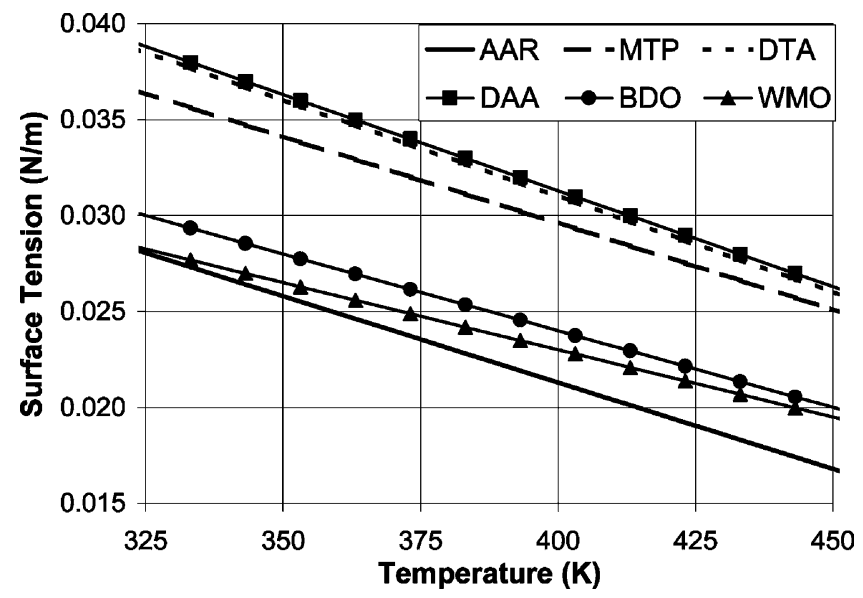

Fig. 7. Variation of surface tension with temperature for the six HTFs. 
tension with temperature for the six HTFs. Additionally, Table 1 provides the correlation equations and the error of correlation predictions.

\section{Data analysis}

Atomization is the process by which aerosol is produced. Here, atomization is induced by forcing the liquid through a small orifice to emulate a leak in a process system. The liquid stream upon leaving the orifice is destabilized by the friction forces between the air and the liquid surface. These aerodynamic forces cause disturbances in the surface of the stream. If these disturbances are large enough to overcome the surface energy of the stream, the stream breaks up. This break-up results in the formation of non-spherical segments known as ligaments, which break-up further until they are small enough to form stable spherical droplets.

The atomization process is easily distinguishable into three zones as indicated in Fig. 8: $\mathrm{AB}$ represents the compact stream, $\mathrm{BC}$ the disintegration zone, and $\mathrm{CD}$ the fully atomized zone. Stream atomization can be characterized by the dependence of droplet diameter on the droplet Weber number, $W e_{D}$, which relates the shear forces that contribute to the stream breakup to the surface tension forces holding the stream together. $W e_{D}=\frac{\rho_{G} V^{2} D}{\sigma}$, where $\rho_{G}$ is the air density, $V$ is the initial velocity of the exiting liquid stream, $D$ is the droplet diameter, and $\sigma$ is the fluid surface tension (Ohnesorge, 1936). $V$ was determined as a function of the pressure drop across the orifice $(\Delta P)$ and the coefficient of discharge $\left(C_{D}\right)$ of the orifice, both of which were measured, as well as liquid density $\left(\rho_{L}\right)$.

$V=C_{D} \sqrt{\frac{2 \Delta P}{\rho_{L}}}$

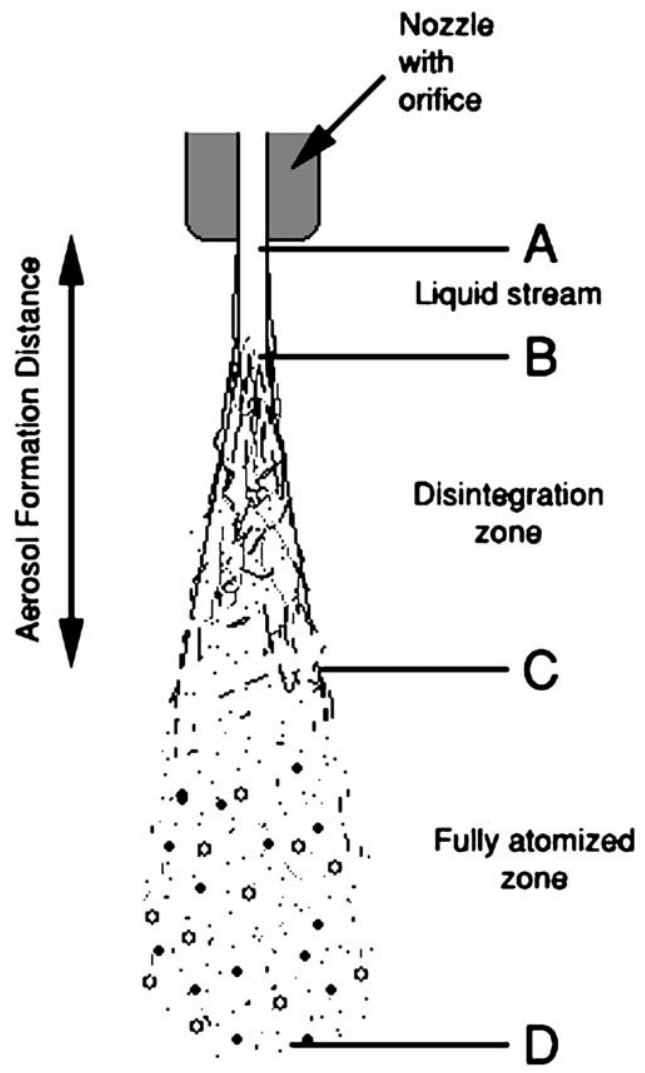

Fig. 8. Atomization of a liquid stream.

High Weber numbers indicate the dominance of shear forces over surface tension forces such that the stream is unstable and is breaking up. Low Weber numbers indicate that surface tension forces dominate, and the stream is stable because either the velocity is too low to induce breakup or much of the stream has already atomized into stable droplets (CD). The critical Weber number range between the stable steam or droplet region and the atomization region was estimated to be $12-22$ by Johnson and Woodward (1998). This range is consistent with

Table 1

Fluid property — temperature $(\mathrm{K})$ correlations

\begin{tabular}{|c|c|c|c|c|c|c|c|c|c|c|}
\hline \multirow{3}{*}{$\begin{array}{l}\text { Heat transfer } \\
\text { fluid }\end{array}$} & \multicolumn{3}{|c|}{ Density $\left(\mathrm{kg} / \mathrm{m}^{3}\right)$} & \multicolumn{4}{|c|}{ Viscosity (Pa s) } & \multicolumn{3}{|c|}{ Surface tension $(\mathrm{N} / \mathrm{m})$} \\
\hline & \multicolumn{3}{|c|}{$\rho=a(T)+b$} & \multicolumn{4}{|c|}{$\mu=x(T)^{y}+z$} & \multicolumn{3}{|c|}{$\sigma=m(T)+n$} \\
\hline & $a$ & $b$ & Error, $\%^{\mathrm{a}}$ & $x$ & $y$ & $z$ & Error, $\%^{\mathrm{a}}$ & $m$ & $n$ & Error, $\%^{\mathrm{a}}$ \\
\hline AAR & -0.6544 & 1073.3 & $-0.1,+0.2$ & $2 \mathrm{e}^{15}$ & -6.9315 & 0.0001 & $-8.0,+3.0$ & -0.00009 & 0.0573 & $-6.8,-1.5$ \\
\hline MTP & -0.6857 & 1209.4 & \pm 0.06 & $7 \mathrm{e}^{14}$ & -6.7336 & 0.0 & \pm 5.8 & -0.00009 & 0.0656 & $-2.8,+3.6$ \\
\hline DTA & -0.7748 & 1274.0 & 0.00 & $8 \mathrm{e}^{11}$ & -5.6838 & -0.0001 & $-7.0,+3.0$ & -0.0001 & 0.0710 & $-3.9,+0.7$ \\
\hline DAA & -0.7103 & 1237.7 & \pm 0.05 & $3 e^{13}$ & -6.2399 & 0.00009 & $-9.5,+2.5$ & -0.0001 & 0.0713 & $-1.0,0.0$ \\
\hline BDO & -0.8379 & 1311.1 & \pm 0.05 & $2 \mathrm{e}^{10}$ & -5.1838 & 0.00007 & $-8.5,+2.2$ & -0.00008 & 0.056 & $-4.3,+0.4$ \\
\hline WMO & -0.4169 & 994.15 & $-0.1,+0.2$ & $8 \mathrm{e}^{11}$ & -5.6182 & 0.0 & $-2.9,+1.9$ & -0.00007 & 0.051 & $-6.8,+2.9$ \\
\hline
\end{tabular}

\footnotetext{
${ }^{a}$ Error, $\%=\frac{\text { predicted value }- \text { actual value }}{\text { actual value }} \times 100$
} 
the data, as shown in Fig. 9, where the critical Weber number determined from measurements is between 11 and 15 . The maximum estimated uncertainty associated with the droplet Weber number is $\pm 10.5 \%$

With the data following established trends, the next step involves predictive correlations relating the mean aerosol droplet diameter to the injection conditions and fluid properties. Modeling of the atomization process is very important in fuel combustion, where the fuel is generally sprayed before it is ignited to increase the combustion efficiency. A vast amount of research in this regard has confirmed that droplet diameter is the most important parameter of combustion efficiency. To circumvent the fact that no theory could completely and accurately describe the atomization process, various methods were adopted to describe quantitatively the atomization process. Dimensional analysis is the most popular quantitative method used, because the use of dimensionless groups decreases the number of experiments required to obtain an empirical expression. The resulting expression is based on experimental data and is therefore readily applicable to the system. Elkotb (1982), Park, Lee and Kihm (1996); Bayvel and Orzechowski (1993), and Kihm and Chigier (1991) have provided analyses of the important parameters to characterize the atomization process.

The operating conditions, temperature and pressure, and the orifice diameter are the parameters that must be related to the mean aerosol droplet diameter. The temperature mainly affects the physical properties of the fluid, which in turn affect the atomization process. Pressure however has a direct influence on the atomization

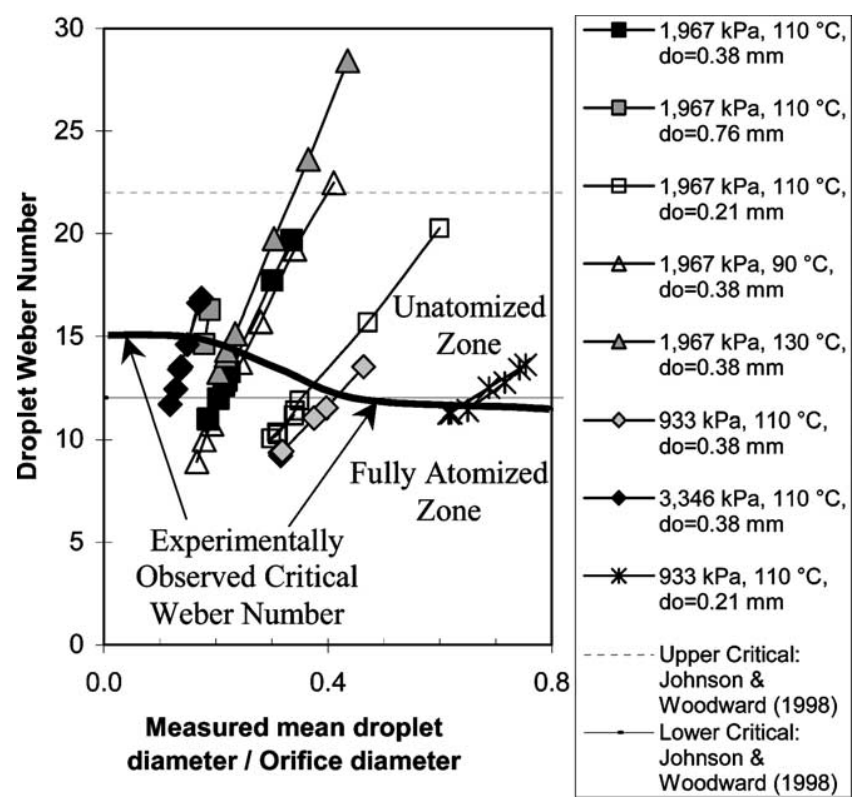

Fig. 9. Analysis of droplet Weber number vs. measured mean droplet diameter/orifice diameter for WMO (white mineral oil) at various injecting pressures, temperatures, and orifice diameters $\left(d_{0}\right)$. process. Higher pressures result in higher spray velocities and an increased shear at the liquid-air interface, which magnifies the instabilities on the liquid stream, causing a more rapid and effective atomization.

Parameters that are important to the atomization process are: $D$ : Aerosol mean droplet diameter; $d_{0}$ : Orifice diameter; $X$ : Axial distance from the orifice; $V$ : Initial velocity of the exiting liquid stream; $\sigma$ : Liquid surface

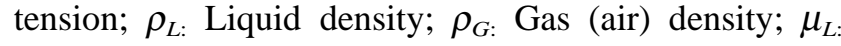

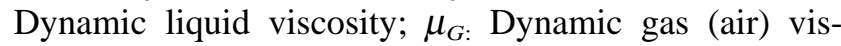
cosity.

The mean droplet diameter used in this study is the Sauter Mean Diameter (SMD), which is the diameter of a uniform set of equivalent droplets with the same total volume and the surface of all droplets as in the real set.

$D=S M D=\frac{\sum D_{n}^{3} \Delta n}{\sum D_{n}^{2} \Delta n}$

where $\Delta n$ is the number of droplets of diameter $D_{n}$.

The basis of dimensional analysis is the selection of the dimensionless parameters by combining the above parameters (Bayvel \& Orzechowsky, 1993).

$D, d_{0}, X, V, s, \rho_{L}, \rho_{G}, \mu_{L}, \mu_{G}$

All of these parameters can be described by a maximum of three units: $\mathrm{M}$ (mass), $\mathrm{L}$ (length), and $\mathrm{T}$ (time). From this list of eight parameters, we designate three parameters, $d_{0}, V$, and $\rho_{G}$, as basic parameters, which contain all three units among them. The next step is to group each of the five remaining parameters with all three basic parameters raised to unknown powers. Each of these groups is a dimensionless parameter, which is determined from the powers on the basic parameters. A function, $f$, which includes all the dimensionless parameters, is then

$f\left(\Pi_{1}, \Pi_{2}, \Pi_{3}, \Pi_{4}, \Pi_{5}, \Pi_{6}\right)=0$

where

$$
\begin{aligned}
& \Pi_{1}=D d_{0}^{a 1} V^{b 1} \rho_{G}^{c 1} \\
& \Pi_{2}=\sigma d_{0}^{a 2} V^{b 2} \rho_{G}^{c 2} \\
& \Pi_{3}=\mu_{L} d_{0}^{a 3} V^{b 3} \rho_{G}^{c 3} \\
& \Pi_{4}=\mu_{G} d_{0}^{a 4} V^{b 4} \rho_{G}^{c 4} \\
& \Pi_{5}=\rho_{L} d_{0}^{a 5} V^{b 5} \rho_{G}^{c 5} \\
& \Pi_{6}=X d_{0}^{a 6} V^{b 6} \rho_{G}^{c 6}
\end{aligned}
$$

The condition that will make each $\Pi$ dimensionless is for the right hand side of Eqs. (3)-(8) also to be dimensionless. From this criterion, the powers can be determined. Examining Eq. (3) for unit L: $0=1+\mathrm{a} 1+$ $\mathrm{b} 1+\mathrm{c} 1$; for unit $\mathrm{M}: 0=\mathrm{c} 1$; for unit $\mathrm{T}: 0=-\mathrm{b} 1$. Therefore, $\mathrm{a} 1=-1 ; \mathrm{b} 1=0 ; \mathrm{c} 1=0$, and hence $\Pi_{1}=$ 
$\mathrm{D} d_{0}^{-1}$. A similar analysis for the other dimensionless parameters yields the complete set of criteria.

$\Pi_{1}=\frac{D}{d_{0}}$

$\Pi_{2}=\frac{\sigma}{\rho_{G} V^{2} d_{0}}$

$\Pi_{3}=\frac{\mu_{L}}{\rho_{G} V d_{0}}$

$\Pi_{4}=\frac{\mu_{G}}{\rho_{G} V d_{0}}$

$\Pi_{5}=\frac{\rho_{L}}{\rho_{G}}$

$\Pi_{6}=\frac{X}{d_{0}}$

We can replace $\Pi_{3}$ by $\Pi_{3}^{\prime}$ :

$\Pi_{3}^{\prime}=\frac{\Pi_{3}^{2}}{\Pi_{2} \Pi_{5}}=\frac{\mu_{L}^{2}}{\rho_{L} \sigma d_{0}}$

and $\Pi_{4}$ by $\Pi_{4}^{\prime}$ :

$\Pi^{\prime}{ }_{4}=\frac{\Pi_{3}}{\Pi_{4}}=\frac{\mu_{L}}{\mu_{G}}$

Substituting these values, eq. (2) becomes

$\frac{D}{d_{0}}=f\left(\frac{\rho_{G} V^{2} d_{0}}{\sigma}, \frac{\rho_{L} \sigma d_{0}}{\mu_{L}^{2}}, \frac{\mu_{L}}{\mu_{G}}, \frac{\rho_{L}}{\rho_{G}}, \frac{X}{d_{0}}\right)$

or

$\frac{D}{d_{0}}=f\left(W e, L p, \frac{\mu_{L}}{\mu_{G}}, \frac{\rho_{L}}{\rho_{G}}, \frac{X}{d_{0}}\right)$

\subsection{Physical significance}

The Weber number $(W e)$ represents the effect of external forces on the droplet development and stream break up and is the ratio of the dynamic forces, contributed by the ambient air, to the surface tension. A higher Weber number indicates a dominance of the dynamic forces with break up of the stream.

$W e=\frac{\rho_{G} V^{2} d_{0}}{\sigma}$

The Laplace number $(L p)$ represents the contribution of the liquid properties to the atomization process and is the ratio of the surface tension forces to the viscous forces within the liquid.

$L p=\frac{\rho_{L} \sigma d_{0}}{\mu_{L}^{2}}$
The density ratio, $\left(\frac{\rho_{L}}{\rho_{G}}\right)$, denotes the ratio of the liquid density to the air density, while the viscosity ratio, $\left(\frac{\mu_{L}}{\mu_{G}}\right)$, denotes the ratio of the liquid viscosity to the air viscosity. In addition, the spatial factor is represented as the normalized length scale, $\left(\frac{X}{d_{0}}\right)$. Also, implicit within the array of parameters mentioned here is the Reynolds number $(R e)$, which represents the ratio of the liquid inertial forces to the viscous forces.

$R e=\frac{\rho_{L} d_{0} V}{\mu_{L}}$

The following relationship holds for the Reynolds number:

$R e=\sqrt{W e \cdot L p \cdot \frac{\rho_{L}}{\rho_{G}}}$

Therefore, we may also use $R e$ instead of $L p$ as a term in the dimensionless equation.

\subsection{Correlations and predictions}

The correlations for all fluids show good agreement with the data. The coefficient of multiple regression was above $80 \%$ for the mean droplet diameters and most of the values predicted from the correlation are within the total measurement uncertainty, as shown in Fig. 10.

The correlation validation is performed on the basis of the basic parameters that are described by the dimensionless groups. All of the obtained equations are expanded in terms of the basic parameters, and the exponent on each parameter is examined for its significance.

$\frac{D}{d_{0}}=A(W e)^{B}(\operatorname{Re})^{C}\left(\frac{\mu_{L}}{\mu_{G}}\right)^{D}\left(\frac{\rho_{L}}{\rho_{G}}\right)^{E}\left(\frac{X}{d_{0}}\right)^{F}$

$D=a\left(\rho_{G}\right)^{b}\left(\mu_{G}\right)^{c}\left(\rho_{L}\right)^{d}\left(\mu_{L}\right)^{e}(\sigma)^{f}(V)^{g}(X)^{h}\left(d_{0}\right)^{i}$

The correlation parameters for the above two equations, using the multiple regression technique, are tabulated for the six fluids tested in Tables 2 and 3. The exponent for liquid viscosity is positive, which means that higher viscosities result in larger aerosol mean droplet diameters and also larger aerosol formation distances. Therefore, while a higher viscosity hinders atomization, under conditions where atomization does occur, the mean droplet diameters will be larger.

The exponent for surface tension is positive, which indicates that, while higher surface tension can produce larger droplets, it also hinders aerosol formation (Tabata, Arai \& Hiroyasu, 1985). All exponents of liquid density are negative, which means that more dense fluids will 

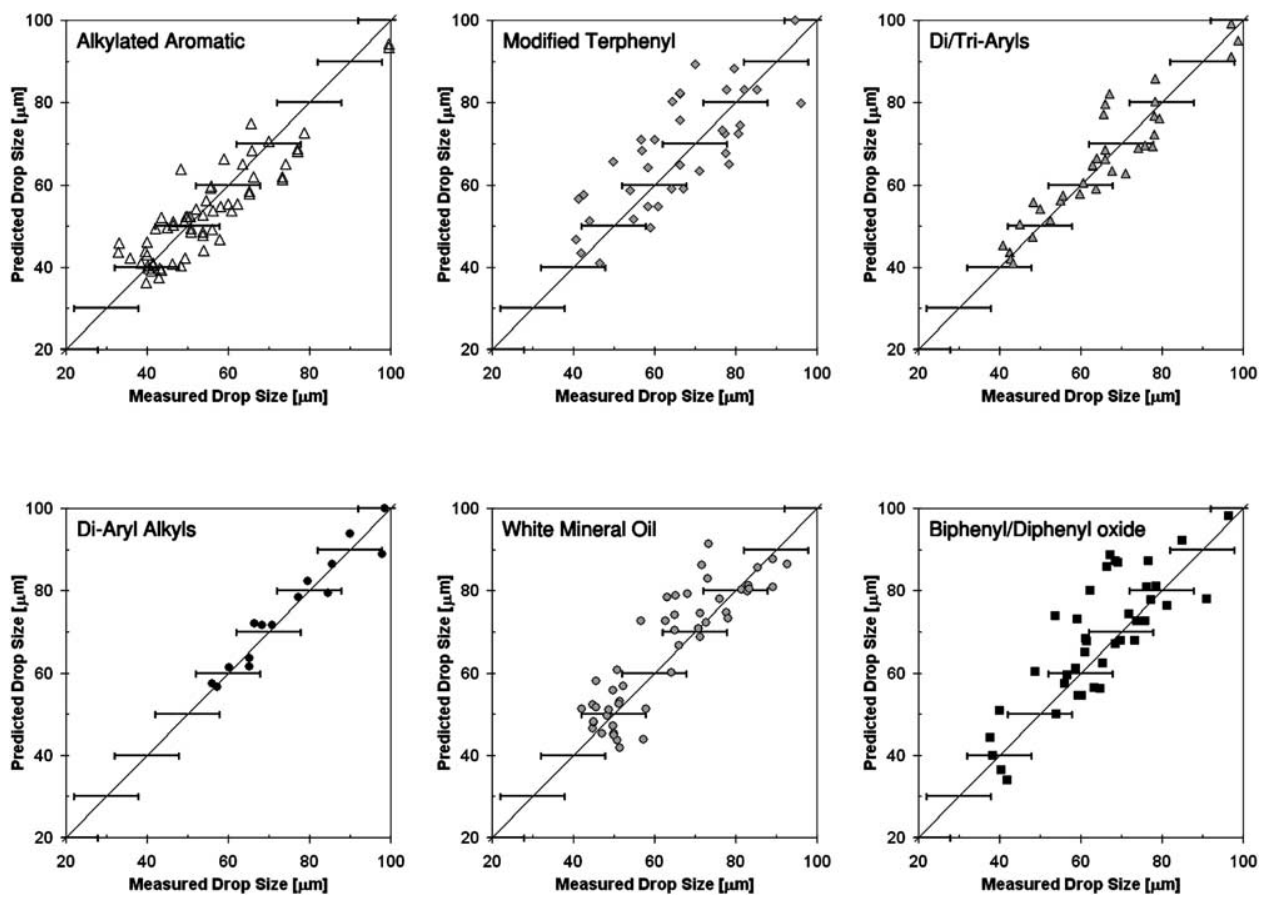

Fig. 10. Correlation results: Predicted mean droplet diameters for six tested fluids (x-error bars indicate $\pm 8 \mu \mathrm{m}$ ).

Table 2

Correlation parameters for six heat transfer coefficients based on eq. (23)

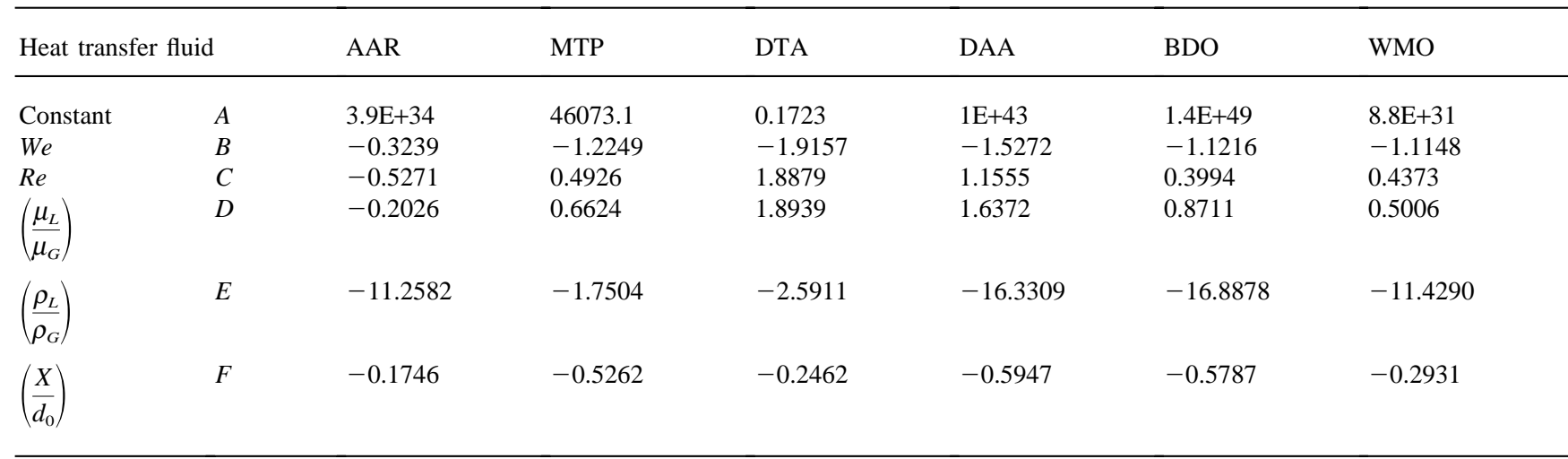

Table 3

Correlation parameters for six heat transfer coefficients based on eq. (24)

\begin{tabular}{|c|c|c|c|c|c|c|c|}
\hline \multicolumn{2}{|c|}{ Heat transfer fluid } & \multirow{2}{*}{$\begin{array}{l}\text { AAR } \\
-11.7853\end{array}$} & \multirow{2}{*}{$\begin{array}{l}\text { MTP } \\
-1.2578\end{array}$} & \multirow{2}{*}{$\begin{array}{l}\text { DTA } \\
-0.7032\end{array}$} & \multirow{2}{*}{$\begin{array}{l}\text { DAA } \\
-15.1755\end{array}$} & \multirow{2}{*}{$\begin{array}{l}\text { BDO } \\
-16.4884\end{array}$} & \multirow{2}{*}{$\begin{array}{l}\text { WMO } \\
-10.9918\end{array}$} \\
\hline Liquid & Density $(d)$ & & & & & & \\
\hline & Viscosity $(e)$ & 0.3245 & 0.1698 & 0.0060 & 0.4818 & 0.4716 & 0.0633 \\
\hline & Surface tension $(f)$ & 0.3239 & 1.2249 & 1.9157 & 1.5272 & 1.1216 & 1.1148 \\
\hline \multirow[t]{2}{*}{ Nozzle } & Velocity $(g)$ & -1.1750 & -1.9572 & -1.9436 & -1.8989 & -1.8437 & -1.7923 \\
\hline & Orifice diameter $(i)$ & 0.3235 & 0.7939 & 1.2184 & 1.2230 & 0.8565 & 0.6156 \\
\hline \multicolumn{2}{|c|}{ Distance from the orifice $(h)$} & -0.1746 & -0.5262 & -0.2462 & -0.5947 & -0.5787 & -0.2931 \\
\hline
\end{tabular}

produce smaller droplets while atomizing much closer to the orifice. This behavior can be rationalized by the fact that denser liquids have a higher kinetic energy and consequently smaller droplets develop. Liquid velocity always has a negative exponent. Higher velocities are caused by higher injection pressures and result in smaller droplets as well as shorter aerosol formation distances (Tabata et al., 1985). The smaller orifice size constricts 
the liquid stream to a greater extent, resulting in smaller droplets and hence confirms the positive exponent. These correlations have a good agreement with experiment and their implications can be rationalized by theory.

\section{Conclusions}

This research has characterized the dependence of aerosol droplet diameter distributions on the operating conditions and the leak size. The most important conclusion for process safety is that significant quantities of aerosol are formed from HTFs at conditions well below their flash points. However, there are threshold conditions below which significant amounts of aerosols were not formed in the tested ranges (Sukmarg et al., 2002). Operating conditions and leak size also had a significant effect on the mean droplet diameter, atomization distance, and the amount of aerosol generated.

These correlations help define a source term for leaking fluids forming aerosols. During the design process the engineer must consider the following additional design criteria:

- The HTF with the higher density will form smaller droplets on leaking.

- The HTF with the higher viscosity is less likely to form aerosol.

- The HTF with the higher surface tension will form larger droplets on leaking.

- Higher operating pressures will produce aerosols closer to the leak and smaller mean droplet diameters.

HTF selection can be based on liquids that are less likely to form aerosols. Design criteria can also incorporate the data to arrive at operating conditions that are less likely to produce aerosols.

Using the correlation described here to define source terms for leaks forming aerosols will help optimize existing dispersion models by relating the dispersion to the operating conditions of the process. Finally, the study of aerosol combustion as a function of drop size distributions, concentrations, and fluid properties will facilitate the development of correlations for the upper and lower explosive limits of HTF aerosol/air mixtures. Such studies are currently being planned and will provide a better understanding of aerosol hazards. This information will make possible a more comprehensive understanding of aerosol explosions and thereby improve process safety.

\section{Acknowledgements}

This aerosol research is supported by the Mary Kay O'Connor Process Safety Center, the Texas Higher Education Coordinating Board through the Advanced Technology Program, and the following industrial sponsors: Ashland Inc., Solutia Inc., Quest Consultants Inc., Conoco Inc., Dow Chemical, Shawnee Engineers, and Huntsman Corporation.

\section{References}

Barth, H. G. (1984). In (pp. 135-172). Modern Methods of Particle Size Analysis, New York: Wiley \& Sons.

Bayvel, L., \& Orzechowski, Z. (1993). Liquid Atomization. Washington D.C: Taylor \& Francis.

Bowen, P. J., \& Shirvill, L. C. (1994). Combustion hazards posed by the pressurized atomization of high-flash point liquids. Journal of Loss Prevention in the Process Industries, 7(3), 233-241.

Eichhorn, J. (1955). Careful! Mist can explode. Petroleum Refiner, 34(11), 194-196.

Elkotb, M. M. (1982). Fuel atomization for spray modeling. Progress in Energy and Combustion Science, 8, 61-91.

Febo, H. L., \& Valiulis, J. V. (1995). Heat transfer fluid mist explosion potential an important consideration for users. In AIChE Loss Prevention Symposium, Norwood, ME. Paper 4F.

Johnson, D. W., \& Woodward, J. L. (1998). Release: A Model with Data to Predict Aerosol Rainout in Accidental Releases. New York: Center for Chemical Process Safety, AIChE.

Hecht, E. (2002). Optics (4th ed.). Reading, MA: Addison-Wesley.

Kihm, K. D., \& Chigier, N. (1991). Effect of shock waves on liquid atomization of a two-dimensional airblast atomizer. Atomization and Sprays, 1(1), 113-136.

Kihm, K. D., Terracina, D. P., Payne, S. E., \& Caton, J. A. (1994). Synchronized droplet size measurements for coal-water slurry sprays generated from a high-pressure diesel injection system. Journal of the Institute of Energy, 67, 2-9.

Ohnesorge, W. (1936). Formation of drops by nozzles and the breakup of liquid jets. Zeitschrift fur Angewandte Mathematik und Mechanik, 16, 355-358.

Park, B. K., Lee, J. S., \& Kihm, K. D. (1996). Comparative study of twin-fluid atomization using sonic or supersonic gas jets. Atomization and Sprays, 6, 285-304.

Sukmarg, P., Krishna, K., Rogers, W. J., Kihm, K. D., \& Mannan, M. S. (2002). Non-intrusive characterization of heat transfer fluid aerosol sprays released from an orifice. Journal of Loss Prevention in Process Industries, 15, 19-27.

Tabata, M., Arai, M., \& Hiroyasu, H. (1985). Effect of fuel viscosity and surface tension on diesel spray drops. Proceedings of the ICLASS 85, London, England, (July 8-10), IIB/1/1.

Vincent, G. C., \& Howard, W. B. (1976). Hydrocarbon mist explosions, prevention by explosion suppression, Part I. Chemical Engineering Progress Technical Manual, 10, 43-47. 